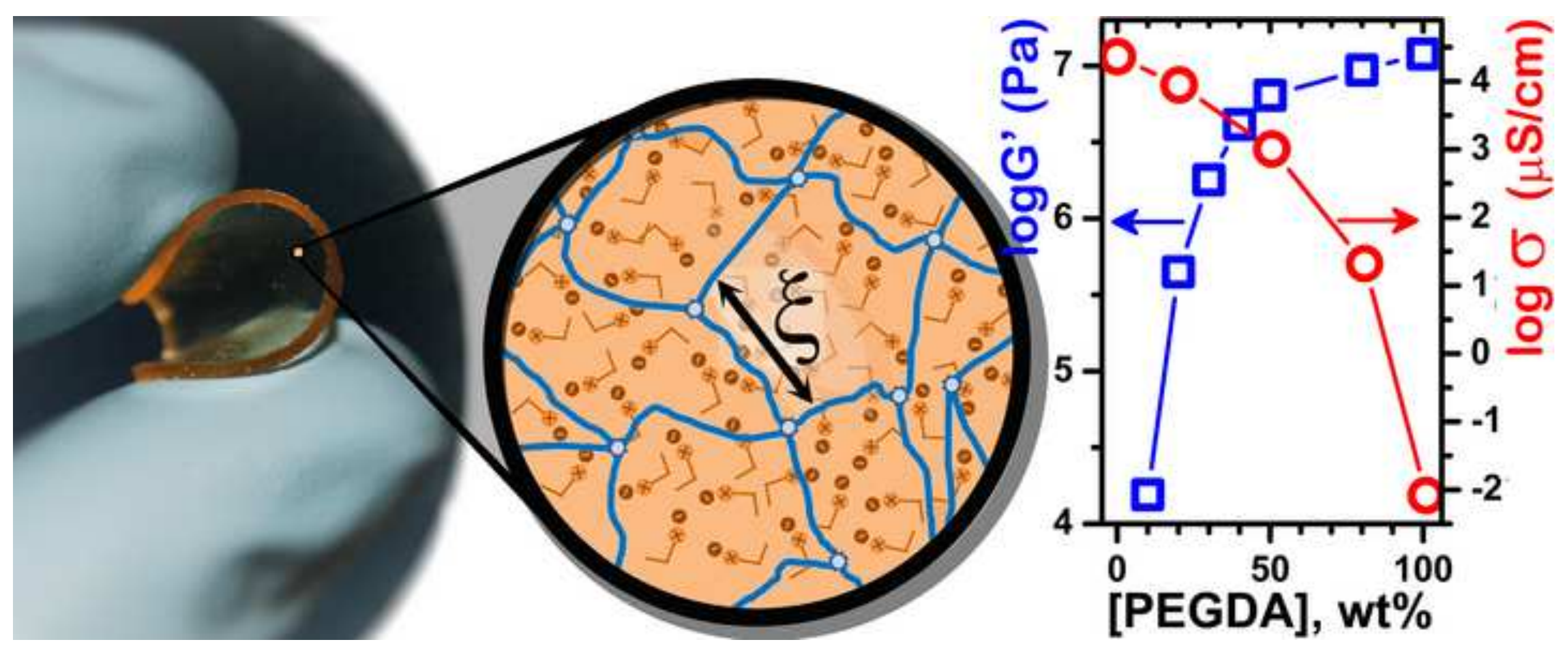




\title{
Mechanical, Dielectric and Structural Characterization of Cross-Linked PEG-Diacrylate/Ethylammonium Nitrate Ionogels
}

\author{
Carlos R. López-Barrón \\ ExxonMobil Chemical Company, Baytown, TX, 77520, USA \\ Peter J. Beltramo \\ Center for Molecular and Engineering Thermodynamics, Department of Chemical and \\ Biomolecular Engineering, University of Delaware, Newark, DE, 19716, USA \\ Yun Liu \\ National Institute of Standards and Technology, Center for Neutron Research, \\ Gaithersburg, Maryland 20899, USA \\ Sung-Min Choi, Min-Jae Lee \\ Department of Nuclear and Quantum Engineering, Korea Advance Institute of Science \\ and Technology, Daejon, Korea
}

\begin{abstract}
We report the preparation and characterization of cross-linked ionogels ( $\mathrm{x}$ IGs) composed of polyethylene glycol diacrylate (PEGDA) and the protic ionic liquid, ethylammonium nitrate (EAN). The cross-linking process has a huge effect on the mechanical properties of the solutions (forming stiff solids from Newtonian solutions) and a minimum penalty on the ionic conductivity. The interdependence of the mechanical and dielectric properties with the network structure of the $\mathrm{x}$-IGs was studied using three experimental probes: torsion and compression mechanical testing, dielectric spectroscopy and small angle neutron scattering. The microstructure, the mechanical strength and the conductivity of the x-IGs depend strongly on the polymer
\end{abstract}

Email address: carlos.r.lopez-barron@exxonmobil.com (Carlos R. López-Barrón)

Preprint submitted to Polymer

January 22, 2016

(C) 2016. This manuscript version is made available under the Elsevier user license http://www.elsevier.com/open-access/userlicense/1.0/ 
concentration and weakly on the temperature. High modulus and relatively low conductivities are associated to small cross-link junction lengths, $\xi$, observed in concentrated samples, whereas large $\xi$ values, observed in dilute samples, result in high conductivities and relatively low modulus. The topological restriction to ionic transport (i.e., to conductivity) is quantified by the obstruction factor, which increases monotonically with $\xi$, while the shear modulus exhibits a power law behavior, $G \sim \xi^{-3}$, in accordance to linear viscoelastic theory.

Keywords: ionogles, solid electrolytes, small-angle neutron scattering

\section{INTRODUCTION}

Ionic liquids (ILs) are salts composed of poorly coordinated ions, which results in low melting temperatures (typically $\mathrm{T}_{m}<100^{\circ} \mathrm{C}$ or $\mathrm{T}_{m}<25^{\circ} \mathrm{C}$ for the case of room temperature ionic, RTIL). These compounds exhibit remarkable properties, such as high stability (both thermal and chemical), low flammability, negligible vapour pressure, and high ionic conductivity [1, $2,3]$. In some instances, however, it is desirable to restrict the fluidity of ILs, while keeping their unique properties for their use in solid electrolytes, and separation membranes or similar devices $[4,5,6,7,8]$. One way to achieve this is by the in situ formation of a three-dimensional network that percolates through the IL [9]. This network imparts solid-like properties to the resulting materials (commonly named ionogels) with minimal decrement of the IL inherent properties.

Ionogels (IGs) can be classified in two broad categories: physical or chemical IGs. The difference between these two lies in the type of cross-linking bonds forming the network. Physical gels are typically formed through weak and reversible interactions (e.g. hydrogen bonding, hydrophobic interactions, etc.). Examples of these type of IGs are those formed by the self-assembly of triblock copolymers with alternated soluble and insoluble polymer blocks $[10,11,12]$. Chemicals IGs involve covalent bonding at the cross-linking junctions. Chemical IGs are of particular interest for the present paper, however a thorough review of both types was recently given by Bideau et al. [9].

The combination of ILs with cross-linked polymers (in-situ or ex-situ) provides important enhancement in mechanical properties, without losing significant conductivity, which are useful in practical applications $[4,13,14$, 15, 16, 17, 18]. Snedden and coworkers [4] synthesized a series of porous 
polymer composites via in situ polymerization and crosslinking of a range of monomers in three N,N-dialkylimidazolium ionic liquids. Susan and coworkers [14] reported the synthesis of ionogels via in situ free radical polymerization of vinyl monomers in the aprotic IL bis(trifluoromethane sulfonyl)imide. This process yielded flexible, transparent IGs with relatively high ionic conductivity $\left(\sim 10^{-2} \mathrm{~S} \mathrm{~cm}^{-1}\right)$. In situ polymerization of methyl methacrylate in the presence of a series of imidazolium ionic liquids was reported by Sterner et al. [18]. They studied the dependence of mechanical properties and AC impedance on the type and content of IL, crosslinker density and molecular weight between crosslinkers. Curing of epoxy resins in presence of ILs is another method to prepare IGs. Matsumoto and Endo [16] synthesized networked polymers by curing a mixture of bisphenol A diglycidyl ether and tetrafunctional epoxy resins with tetraethylenepentamine in presence of the aprotic ionic liquid, 1-ethyl-3-methylimidazolium bis(trifluoromethane sulfonyl)imide. They observe significant IL confinement within the network structure, which is closely related to the ionic conductivity and mechanical strength of these compounds.

Functionalized polyethylene glycol (PEG) compounds have been used as crosslinkers in the synthesis of PEG-based ionogels. Klingshirn and coworkers [13] reported IGs formation via crosslinking of disuccinimidylpropyl PEG monomers with four-arm tetramine PEG crosslinkers disolved in the ionic liquid, 1-hexyl-3-methylimidazolium bis(trifluoromethane sulfonyl)imide. A series of hyperbranched PEGs connected with triazine linkages were used by Tigelaar et al. to prepare highly conductive polymer electrolytes, containing $\mathrm{Li}^{+}$and pyrrolidinium salts of trifluoromethanesulfonimide, via crosslinking reactions [15]. Rupp et al. [17] used ionizing radiation to induce fragmentation and subsequent crosslinking of PEG in the presence of a conducting salt, litium bis (trifluoromethane sulfonyl)imide, and an ionic liquid (N-methylN-butylpyrrolidinium bis(trifluoromethane sulfonyl)imide. By this process they prepared solid films with high ionic conductivity.

As illustrated above, several approaches to prepare chemical IGs can be found in the literature. However, to our knowledge, all of them are based on aprotic ILs (e.g., imidazoliums, pyridiniums, and phosphoniums), and none on a protic IL. In general, the fluidities and attendant conductivities of protic ILs tend to be much higher than those of aprotic ILs $[2,19]$. Here, we describe the formation of $\mathrm{x}$-linked ionogels ( $\mathrm{x}$-IGs) via dissolution of polyetylene glycol diacrylate (PEGDA) in ethylammonium nitrate (EAN, a protic ionic liquid) and the subsequent photopolymerization of PEGDA. Structural, 
mechanical and dielectric properties of the resulting ionogels are probed via small angle neutron scattering, torsion and uniaxial compression mechanical tests and dielectric spectroscopy, respectively. The interrelation between these properties as well as their dependence on temperature and composition is studied.

\section{EXPERIMENTAL SECTION}

Materials. Polyethylene glycol diacrylate (PEGDA, Mn = $575 \mathrm{~g} / \mathrm{mol}$ ) and the photoinitiator, 1-hydroxycyclohexyl phenyl ketone (99\%), were purchased from Sigma-Aldrich and used as received. Ethylammonium nitrate (EAN, $97 \%$ ) was purchased from Iolitec. Deuterated EAN (dEAN) was prepared by mixing equimolar quantities of EAN and $\mathrm{D}_{2} \mathrm{O}$ (Aldrich, $99.9 \%$ ) under nitrogen atmosphere at $40{ }^{\circ} \mathrm{C}$ for $3 \mathrm{hrs}$ and redrying by heating under vacuum for at least $6 \mathrm{hrs}$ at $100{ }^{\circ} \mathrm{C}$. The cycle was repeated three times to achieve $83 \%$ substitution of amino-hydrogens for deuterium, as proved by ${ }^{1} \mathrm{H}$ NMR [20]. The last drying step was carried out for 24 hrs to reduce the water content to $0.3 \mathrm{wt} \%$, as ascertained by Karl-Fisher titration.

Synthesis of PEGDA/dEAN coss-liked ionogels. PEGDA and dEAN are both liquid at room temperature and entirely miscible at all compositions. PEGDA/dEAN mixtures were prepared with compositions in the range: $5 \mathrm{wt} \% \leq[\mathrm{PEGDA}] \leq 100 \mathrm{wt} \%$. 1-hydroxycyclohexyl phenyl ketone

was added to each solution with a composition of $0.1 \mathrm{wt} \%$ with respect to PEGDA mass. PEGDA/dEAN solutions were poured into glass Petri dishes, and exposed to an ultraviolet (UV) source $\left(365 \mathrm{~nm}, 1.35 \mathrm{~mW} / \mathrm{cm}^{2}\right.$ ) for 10 min, to form a percolating three-dimensional network, i.e., x-linked ionogels (x-IGs), by free radical reaction of the two acrylate double-bonds in PEGDA (see Scheme 1). $\mathrm{x}$-IGs with thickness $\sim 3 \mathrm{~mm}$ were prepared for mechanical testing. $\mathrm{x}$-IGs for SANS and dielectric measurements were synthesized within the respective measuring cells, as described below.

Torsion and compression mechanical tests. Torsion tests were performed on a strain controlled rheometer (ARES-G2, TA Instruments) operating in torsion mode, using rectangular samples with dimensions: $9 \mathrm{~mm}$ width, $7.8 \mathrm{~mm}$ long and $3 \mathrm{~mm}$ thick. Frequency sweeps $(0.1$ to $100 \mathrm{rad} / \mathrm{s})$ were performed at temperatures ranging from $25^{\circ} \mathrm{C}$ to $100{ }^{\circ} \mathrm{C}$, with a strain of $0.1 \%$. The software of the rheometer calculates the shear moduli, based 
<smiles>C=CC(=O)OCCOC(C)(C)C(=O)OC(C)=O</smiles><smiles>CCCCCC(C)C(C)N[NH+]([NH])CC</smiles><smiles>CC(CC(C)(C)C)OC(=O)C(C(=O)OCCC(C)(C)C)C(C)(C)C</smiles>

Scheme 1: Schematic representation of the photopolymerization reaction of PEGDA in the presence of ethylammonium nitrate (EAN).

on the torque response and the sample geometry. Axial compression testing were performed on $\mathrm{x}-\mathrm{IG}$ cylinders with $8 \mathrm{~mm}$ in diameter and $3 \mathrm{~mm}$ in height at a linear strain rate of $0.1 \mathrm{~mm} / \mathrm{min}$ using the ARES-G2 rheometer. Only the low strain (Hookean) modulus $(E)$ is of interest for this study. $E$ is calculated by taking the slope of the true stress versus true strain curve, in the low strain region. Both torsion and compression tests were performed by quintuplicate for each sample.

Small angle neutron scattering (SANS) measurements. PEGDA/dEAN solutions were injected in $1 \mathrm{~mm}$ thick titanium cells with quartz windows. The samples were exposed to the UV source through the cell windows for 10 min. Selected samples were prepared without photo-initiator and measured in the liquid state. SANS measurements were performed using the 40 m SANS instrument at the HANARO, the Korea Atomic Energy Research Institute (KAERI) in Daejeon, Republic of Korea. Neutrons of wavelength $\lambda=6 \AA$ with a full width half-maximum $\Delta \lambda / \lambda=12 \%$ were used. Three configurations of sample-to-detector distance of 1.16, 7.78, and $17.9 \mathrm{~m}$ were 
used to cover the $q$ range of $0.003 \AA^{-1}<q<0.6 \AA^{-1}$, where $q=(4 \pi / \lambda)$ $\sin (\theta / 2)$ is the magnitude of the scattering vector and $\theta$ is the scattering angle. Sample scattering was corrected for background and empty cell scattering and the sensitivity of individual detector pixels. The corrected data sets were placed on an absolute scale through the direct beam flux method. All the SANS measurements were carried out at $25^{\circ} \mathrm{C}$ using quartz window cells of $1 \mathrm{~mm}$ path length.

Dielectric spectroscopy. Dielectric spectroscopy measurements were performed using a specially designed parallel plate electrode cell attached to an Agilent 4294A-1D5 impedance analyzer. The dielectric cell, modelled after Ref. 21, consists of a pair of stainless steel electrodes clamped around a $4.24 \mathrm{~mm}$ thick acrylic spacer, which serves as the sample chamber. Liquid samples are loaded with a syringe, taking care to avoid the development of any air bubbles that would alter the spectra. Frequency sweeps are made from $110 \mathrm{MHz}$ to $40 \mathrm{~Hz}$ at a nominal oscillator voltage of $0.5 \mathrm{~V}$. The temperature is controlled to $0.5{ }^{\circ} \mathrm{C}$ by flowing silicone oil through cylindrical bores in the electrodes and monitored in the sample cell with a thermocouple. Open/short compensation is performed to remove stray impedances in parallel and in series with the sample. The corrected impedance spectra, $Z^{*}(\omega)$, is interpreted as a frequency dependent conductivity and dielectric constant as

$$
\frac{1}{Z^{*}(\omega)}=\Gamma\left[\sigma(\omega)+i \omega \varepsilon_{0} \varepsilon^{\prime}(\omega)\right]
$$

where $\Gamma$ is the measured cell constant, $\sigma(\omega)$ is the conductivity, $\omega$ is the angular frequency, $\varepsilon_{0}$ is the permittivity of free space and $\varepsilon^{\prime}(\omega)$ is the dielectric constant. The transparent acrylic sample chamber allowed liquid samples to be loaded and UV-cured for at least 30 minutes within the dielectric cell before making measurements. After disassembling the cell, it was found that the sample cured with minimal deformation inside the sample chamber.

\section{RESULTS AND DISCUSSION}

Mechanical strength of x-IGs. The mechanical properties of PEGDA/dEAN xIGs were measured at different temperatures and compositions. Figure 1 shows the shear elastic modulus measured in torsion mode with rectangular 
geometry (see picture in Figure 1). As shown in Figure 1a, the frequency dependence of the dynamic moduli is negligible. The $G^{\prime}$ values shown in Figure $1 \mathrm{~b}$ correspond to an applied frequency of $1 \mathrm{rad} / \mathrm{s}$ (with $0.1 \%$ strain). The shear modulus shows a strong concentration dependence at $[\mathrm{PEGDA}]<50$ wt $\%$. $G^{\prime}$ increases three orders of magnitude in the range $10 \mathrm{wt} \% \leq$ [PEGDA] $\leq 50 \mathrm{wt} \%$. A weaker concentration effect is observed at higher concentrations.

In general $G^{\prime}$ increases with temperature, as expected from the linear viscoelastic theory [22], which predicts

$$
G=\nu k_{B} T
$$

where $\nu$ is the number density of network strands, $k_{B}$ is the Boltzman constant and $\mathrm{T}$ is the absolute temperature. This effect is more pronounced at higher compositions, as evidenced in the plot given in Figure 1c. This implies that the network density is a function of both temperature and composition. Let us consider the relation between "mesh size" (defined as the distance between neighboring junctions in the macromolecular network), $\xi$, and the shear modulus [23]

$$
G \approx A k_{B} T / \xi^{3}
$$

where the constant $A$ accounts for the details of the network structure. This expression is equivalent to Eqn. 2 (i.e., $\nu \approx A / \xi^{3}$ ). If no break up or formation of junctions occurs upon heating, then $A$ is a function only of the polymer concentration, whereas $\xi$ varies with both [PEGDA] and temperature.

The uniaxial compression modulus was measured at room temperature using disk-shaped specimens of $8 \mathrm{~mm}$ in diameter. The picture in the right hand side of Figure 2 shows an example of these discs mounted in the compression tool. The compressive stress-strain curves for x-IGs with different compositions are shown in Figure 2. The initial modulus (Young modulus, $E$ ), divided by 3 , is shown as a function of polymer concentration in the inset of Figure 2, along with the shear modulus obtained by torsion measurements. This plot shows that the ratio $E / G$ is, overall, slightly lower than the expected value of 3 for small deformations of incompressible materials [24]. However the deviation from $E=3 G$ is within the experimental error bars (also see Table 1). This is not surprising, considering that both moduli were 

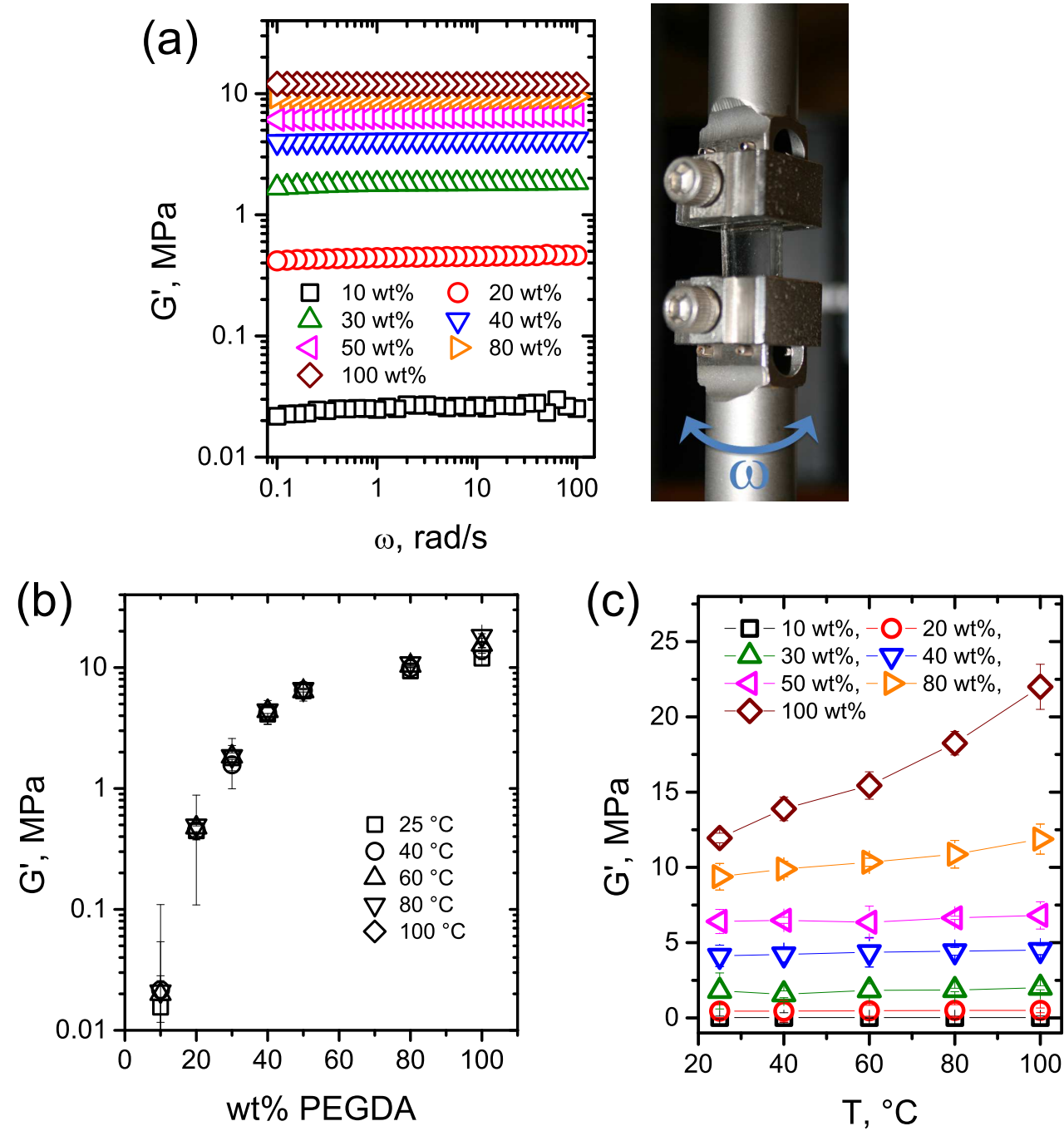

Figure 1: (a) Storage shear modulus $\left(G^{\prime}\right)$ versus frequency for PEGDA/dEAN crosslinked ionogels (x-IGs) with the indicated concentrations, measured at $25{ }^{\circ} \mathrm{C}$. (b) $G^{\prime}$ versus concentration at the indicated temperatures. (c) $G^{\prime}$ as a function of temperature for xIGs with different compositions. The data in (b) and (c) correspond to measurements with frequency of $1 \mathrm{rad} / \mathrm{s}$ and $0.1 \%$ strain. The picture (upper-right) shows a rectangular sample mounted on the torsion tool used for oscillatory tests. 


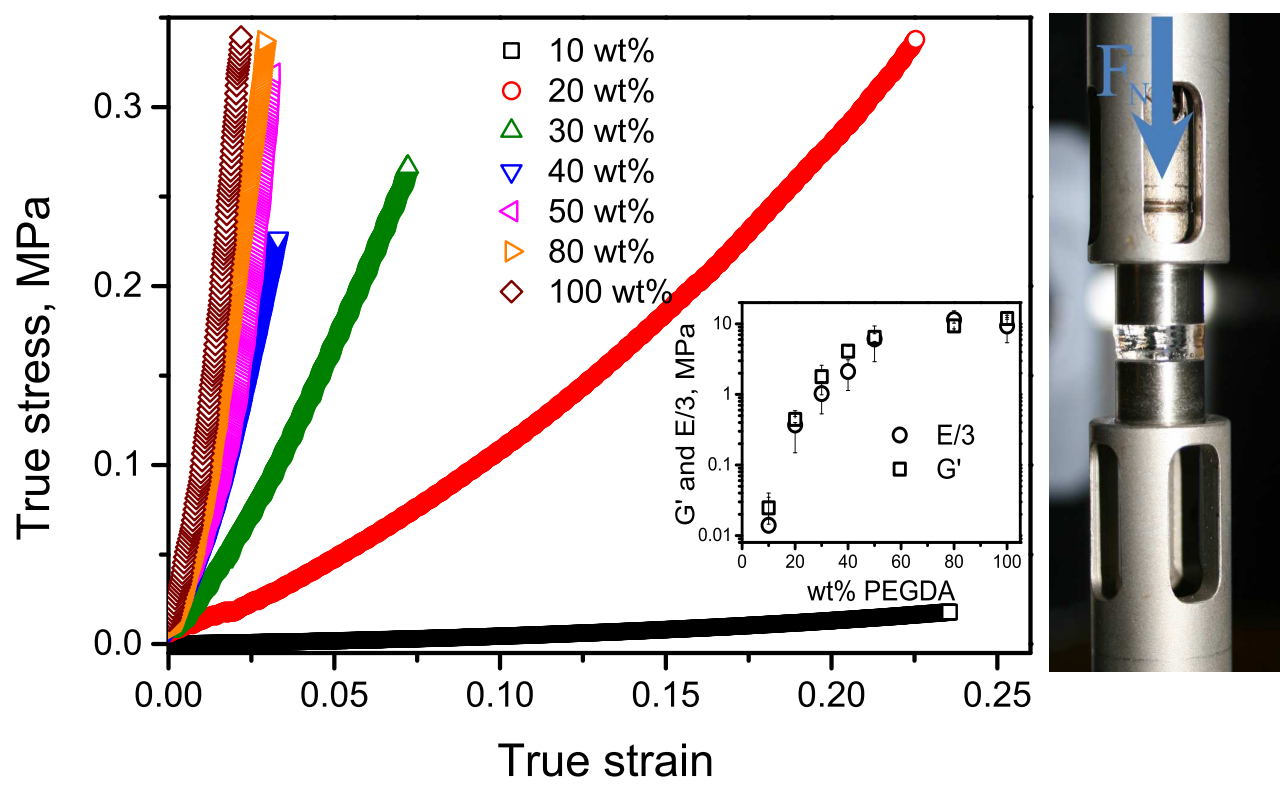

Figure 2: Stress response to compressive strain for PEGDA/dEAN crosslinked ionogels at the compositions indicated, measured at $25{ }^{\circ} \mathrm{C}$. Inset: Storage shear modulus, $\left(G^{\prime}\right)$ measured via dynamic torsion tests (see Figure 1), and the Young modulus $(E)$ divided by 3 , as a function of PEGDA concentration. The picture (right) shows a disc-shaped sample loaded on the tool for compression tests.

measured at low deformation values, i.e., in the linear elasticity regime.

Structural analysis via SANS. SANS measurements were performed on PEGDA/dEAN solutions and $\mathrm{x}$-linked ionogels with compositions ranging from $5 \mathrm{wt} \%$ to $60 \mathrm{wt} \%$ at room temperature. Figure 3 show circularlyaveraged SANS spectrum for samples with different compositions. The inset shows a comparison between samples before and after x-linking for $10 \mathrm{wt} \%$ and $20 \mathrm{wt} \%$ samples. Clearly the formation of a three-dimensional network is marked by an increase in the scattering intensity at low q-values, whereas the negligible scattering observed in the solution (before $\mathrm{x}$-linking) samples indicate the absence of microstructural features, i.e., perfect mixing in a molecular scale.

SANS profiles of $\mathrm{x}$-linked samples (Figure 3) consist of a single broad shoulder, which is typical for polymer solutions and randomly crosslinked hydrogels [26]. The SANS profiles can be fit with the generic Lorentzian 


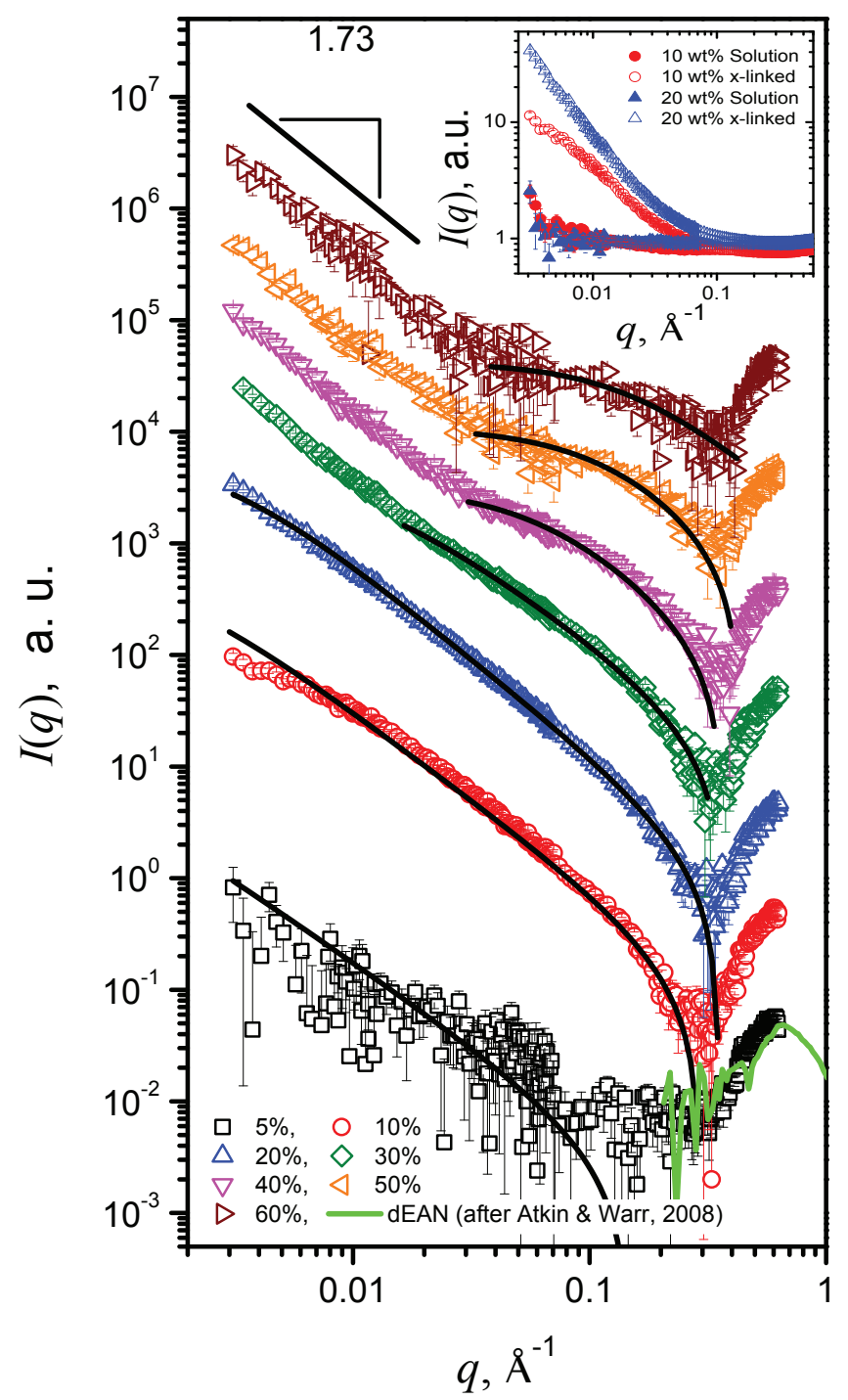

Figure 3: SANS spectra for PEGDA/dEAN crosslinked ionogels with PEGDA concentrations indicated. Solid (black) lines are fits to Eqn 4. The green line superimposed to the $5 \%$ sample data at $q>0.2 \AA^{-1}$ corresponds to SANS from pure dEAN, as reported in Ref. 25. The inset shows the SANS spectra for $10 \mathrm{wt} \%$ and $20 \mathrm{wt} \%$ solutions before and after crosslinking. All SANS measurements were carried out at $25^{\circ} \mathrm{C}$. 
Table 1: Mechanical, structural and conductivity parameters for PEGDA/dEAN crosslinked ionogels and their components.

\begin{tabular}{|c|c|c|c|c|c|c|c|}
\hline \multirow[b]{2}{*}{$\begin{array}{l}{[\text { PEGDA }]} \\
\text { wt } \%\end{array}$} & \multicolumn{2}{|c|}{ Mechanical tests } & \multicolumn{3}{|c|}{ SANS } & \multicolumn{2}{|c|}{ Conductivity } \\
\hline & $\begin{array}{c}E \\
\mathrm{MPa}\end{array}$ & $\begin{array}{c}G^{\prime} \\
\mathrm{MPa}\end{array}$ & $\begin{array}{c}\xi \\
\mathrm{nm}\end{array}$ & $m$ & $A$ & $\begin{array}{c}E_{a} \\
\mathrm{~kJ} / \mathrm{mol}\end{array}$ & $\begin{array}{c}\sigma_{0} \\
\mu \mathrm{S} / \mathrm{cm}\end{array}$ \\
\hline $0(\mathrm{dEAN})$ & -- & -- & -- & -- & -- & $20.4 \pm 0.30$ & $8.93 \times 10^{7}$ \\
\hline 5 & -- & -- & $265 \pm 32$ & 1.45 & -- & - & -- \\
\hline 10 & $0.047 \pm 0.02$ & $0.025 \pm 0.01$ & $70.3 \pm 21$ & 1.58 & 2180 & - & -- \\
\hline 20 & $1.23 \pm 0.22$ & $0.443 \pm 0.04$ & $37.1 \pm 13$ & 1.67 & 5540 & $24.7 \pm 0.56$ & $1.97 \times 10^{8}$ \\
\hline 30 & $3.43 \pm 0.50$ & $1.79 \pm 0.80$ & $9.90 \pm 4.0$ & 1.63 & 423 & - & -- \\
\hline 40 & $7.05 \pm 0.98$ & $4.11 \pm 0.71$ & $2.62 \pm 0.56$ & 1.68 & 18.3 & - & -- \\
\hline 50 & $20.4 \pm 3.21$ & $6.44 \pm 0.84$ & $1.23 \pm 0.29$ & 1.65 & 2.89 & $41.5 \pm 1.25$ & $1.81 \times 10^{10}$ \\
\hline 60 & -- & -- & $1.04 \pm 0.18$ & 1.76 & -- & - & -- \\
\hline 80 & $38.2 \pm 3.80$ & $9.36 \pm 0.88$ & -- & -- & -- & $59.6 \pm 0.71$ & $6.44 \times 10^{11}$ \\
\hline 100 (PEGDA) & $31.1 \pm 3.91$ & $11.9 \pm 0.52$ & -- & -- & -- & $63.2 \pm 2.47$ & $9.98 \times 10^{8}$ \\
\hline
\end{tabular}

model

$$
I(q)=\frac{I_{0}}{1+(\xi q)^{m}}
$$

where $I$ is the scattering intensity, $q$ is the scattering vector, $\xi$ is the correlation length, $m$ is the Porod exponent, and $I_{0}$ is a $q$-independent fitting constant. In a network swollen with a good solvent, the correlation length, $\xi$, gives the mesh size and the Porod exponent, $m$, gives the fractal dimension of the scattering object, $d_{f}$, which is essentially the inverse of the scaling exponent, $\kappa$, associated to the polymer chain conformation [26]. For a polymer chain that behaves as a random walk or as an ideal Gaussian chain, $\kappa=0.5$ (i.e., $d_{f}=2$ ). For highly swollen polymer chains, a self-avoiding random walk (with $\kappa=0.588, d_{f}=1.7$ ) is a better model [27]. Solid lines in Figure 3 are fits to Eqn. 4, using the parameter given in Table 1. From the fitted values of the fractal dimension, it is clear that the polymer chains between network junctions are highly extended for all the x-IGs interrogated with SANS. Interestingly, the network mesh size $(\xi)$ shows a strong dependence on the composition at $[\mathrm{PEGDA}]>5 \mathrm{wt} \%$, and reaches a plateau at high com- 


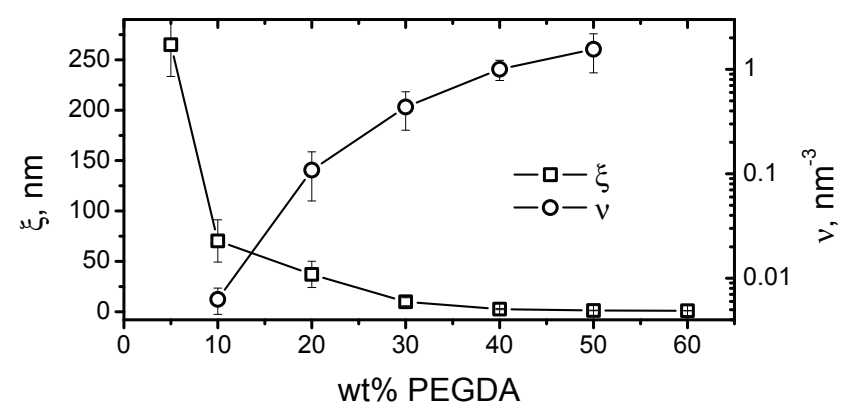

Figure 4: Correlation length $(\xi)$ and number density of network strands $(\nu)$ as a function of concentration for PEGDA/dEAN crosslinked ionogels at $25^{\circ} \mathrm{C}$.

positions (see Figure 4). Moreover, the degree of swelling is a strong function of the composition at $[\mathrm{PEGDA}]<0.5 \mathrm{wt} \%$ and seems to reach a plateau at high compositions (see Figure 4).

The intensity upturn observed in the SANS profiles (Figure 3) at $q>0.3$ $\AA^{-1}$ correspond to the nanoscale segregation of EAN [25]. Atkin and Warr recently reported a scattering peak at $q \sim 0.65 \AA^{-1}$ for pure dEAN, corresponding to Bragg spacing of $\sim 9.7 \AA$, which, along with the fact that EAN is optically isotropic, led them to conclude that EAN form a locally sponge-like structure [25]. Their data is shown as a solid (green) line superimposed to the scattering data of the $5 \%$ solution, in the high-q region (Figure 3). The "EAN peak" is common for all the profiles, which indicates that the EAN "sponge-like" structure remains regardless of the formation of the PEGDA network structure, at least at compositions $[\mathrm{PEGDA}] \leq 60 \mathrm{wt} \%$. On the other hand, a scattering upturn at low $q$ values becomes evident at compositions $[\mathrm{PEGDA}] \geq 30 \mathrm{wt} \%$. This upturn, which is described in Figure 3 by a power law with exponent $\sim-1.73$, could be due to the formation of fractal-like aggregates. Unfortunately, the lack of scattering data at low $q$ values impedes us to elucidate the type and dimensions of such aggregates.

Using the correlation length obtained by SANS, $\xi_{S A N S}$ and Eq. 3, we can estimate the value of the constant $A=\xi_{S A N S}^{3} \cdot G / k_{B} T$. This constant was assumed to be equal to 1 in a recent rheometry study of a triblock copolymer physical gel [28]. We observe a strong dependence of $A$ with concentration (see Table 1). The effective number density of network strands is calculated as $\nu=A / \xi_{S A N S}^{3}$ and plotted in Figure 4 . This x-link density dependence on $[\mathrm{PEGDA}]$ is not unexpected given that, as the concentration increases, 
the functional end groups on PEGDA chains have a higher probability to find each other in the solution and form crosslink points, which results in an overall tighter network.

Dielectric properties of x-IGs. The dielectric behavior of dEAN and PEGDA/dEAN solutions before and after x-linking, at $25{ }^{\circ} \mathrm{C}$, is shown in Figure 5. The upturn of the apparent dielectric constant at low frequencies observed in EAN and the solutions is due to electrode polarization [21, 29]. Hollingsworth and Saville studied the electrode polarization in a dielectric cell, similar to that used here, using aqueous electrolyte solutions [21]. Based on standard eletrokinetic theory and experimental observations, they found that electrode polarization effects follow a simple power law with frequency, $\omega^{-2}$. The same behavior was observed in our samples (see Figure 5a). After x-linking, the ionic transport in the cell is impeded, decreasing the frequency where electrode polarization effects are evident. The conductivity, $\sigma$, decreases as [PEGDA] increases and, similar to the dielectric constant response, a further abatement in $\sigma$ is observed after UV-curing (see Figure 5b). This indicates that the network formation imposes a topological constraint to ionic transport. These differences are less evident in the dilute sample (20 wt \%), where the network is looser.

The conductivity spectra is mostly structureless, with a slight upturn at high frequencies for the PEGDA and 80/20 PEGDA/dEAN sample. The inflection point prior to the upturn marks the characteristic frequency for ionic conduction relaxation, whose reciprocal is the characteristic conduction time constant, or the time to overcome the largest energetic barrier to ion conduction [30]. Note that this relaxation is nearly 100 times faster for the un-crosslinked PEGDA compared to the crosslinked PEGDA (Figure 5b), and roughly 10 times faster at $60^{\circ} \mathrm{C}$ than at $25^{\circ} \mathrm{C}$ (Figure $6 \mathrm{~b}$ ). As previously observed in electrolyte aqueous solution, electrode polarization does not have a drastic effect on the conductivity spectra [21, 29], causing a decrease in $\sigma$ at lower frequencies than in $\epsilon^{\prime}$. The effect of temperature on the dielectric behavior of $\mathrm{x}-\mathrm{IG}$ s is shown in Figure 6. The electrode polarization effect reverts to higher frequencies upon heating to $60{ }^{\circ} \mathrm{C}$, due to a trivial increase in motion of EAN ions [31]. Moreover, the conductivity shows the expected increase with temperature for $\mathrm{dEAN}$ and all the $\mathrm{x}$-IGs. The conductivity as a function of [PEGDA], before and after x-linking, at $25^{\circ} \mathrm{C}$ and $60{ }^{\circ} \mathrm{C}$ are shown in Figure 7. This plot summarizes the decreasing effect of x-linking and the increasing effect of heating on $\sigma$. The conductivity values used 

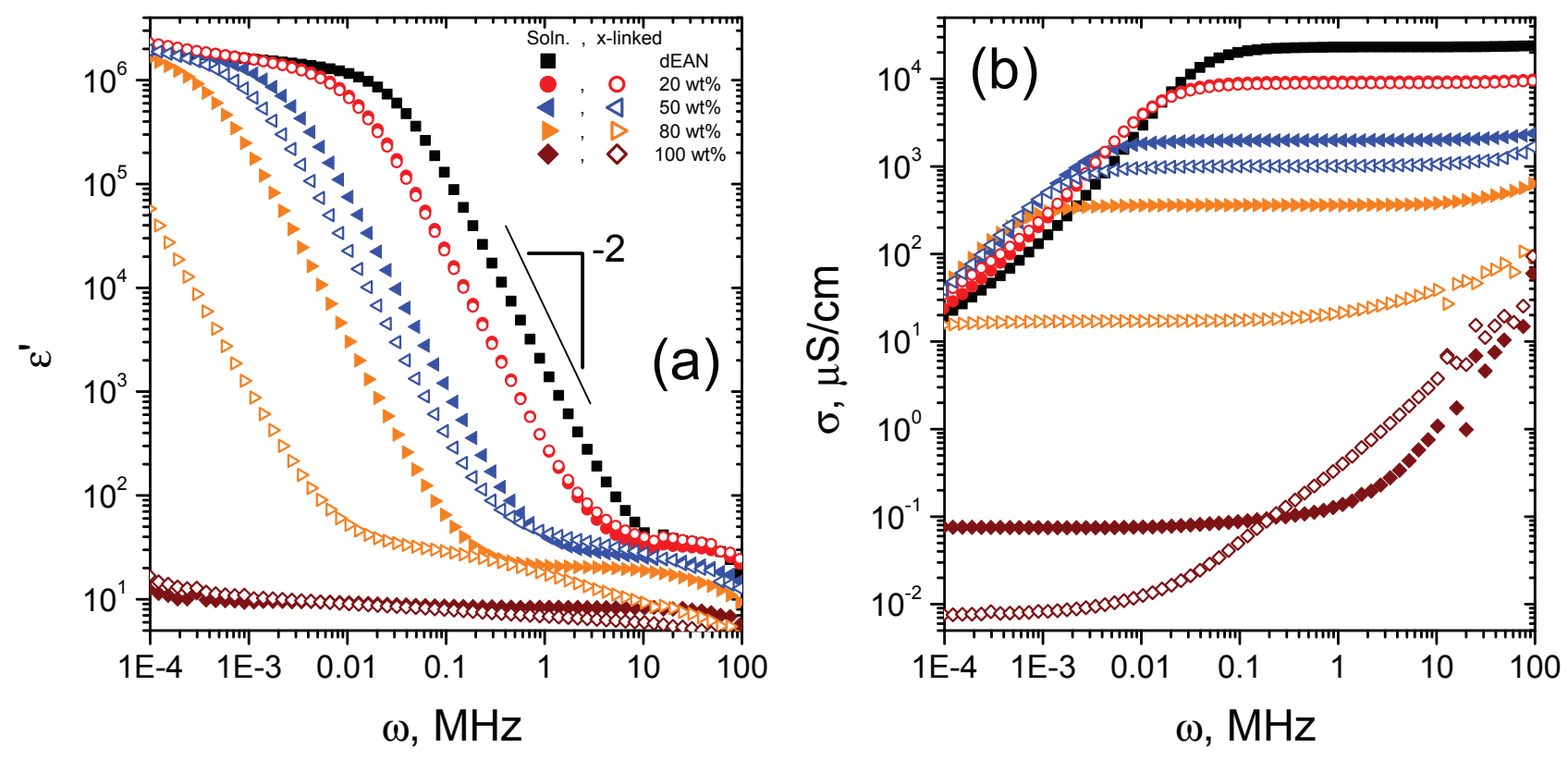

Figure 5: Dielectric response for dEAN and PEGDA/dEAN solutions (before and after crosslinking) as a function of frequency. (a) Apparent dielectric constant, $\epsilon^{\prime}(\omega)$. (b) Apparent conductivity, $\sigma(\omega)$. Symbols in (a) and (b) correspond to the same compositions. All measurements were carried out at $25{ }^{\circ} \mathrm{C}$. 

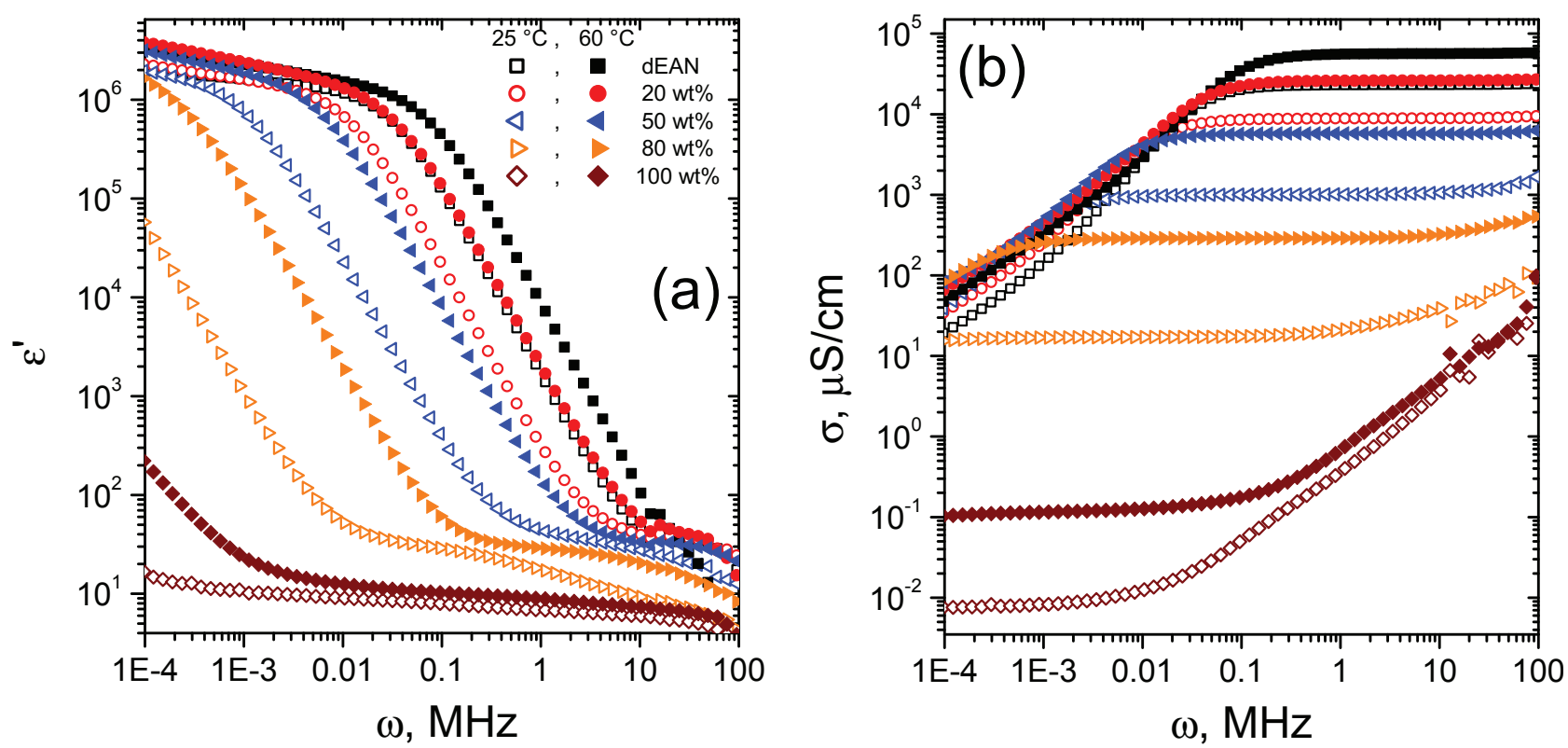

Figure 6: Dielectric response at $25^{\circ} \mathrm{C}$ and $60{ }^{\circ} \mathrm{C}$ for dEAN and PEGDA/dEAN crosslinked ionogels as a function of frequency. (a) Apparent dielectric constant, $\epsilon^{\prime}(\omega)$. (b) Apparent conductivity, $\sigma(\omega)$. Symbols in (a) and (b) correspond to the same compositions.

for all dEAN containing samples were taken at $1 \mathrm{MHz}$ to avoid electrode polarization effect, while the values for PEGDA were taken at $1 \mathrm{kHz}$ to avoid self-inductance effect.

The conductivity was found to follow a simple Arrhenius expression

$$
\sigma=\sigma_{0} \exp [-E a / R T]
$$

for all the samples, in the temperature range studied here. In Eqn. 5, $E_{a}$ is the activation energy for conductivity, $R$ is the gas constant and $\sigma_{0}$ is a temperature-independent constant. Arrhenius plots of the conductivity for several compositions are shown in the inset of Figure 7, along with fits to Eqn. 5 , using the parameters shown in Table 1. The increasing activation energy with [PEGDA] reflects the increasing topological restriction to ion transport by the $\mathrm{x}$-IG network, whose mesh size $(\xi)$ decreases with composition (see Figure 4).

In order to quantify the ionic transport hindrance by the network structure, we calculate the obstruction factor [32] 


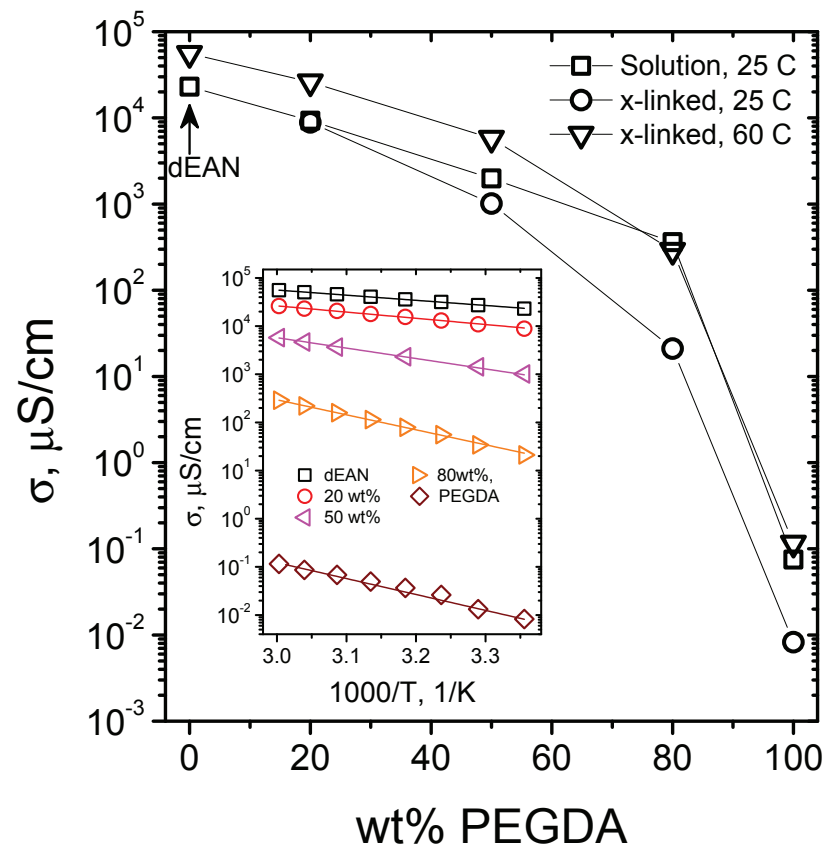

Figure 7: Conductivity as a function of PEGDA for PEGDA/dEAN solutions (before and after crosslinking), measured at $25^{\circ} \mathrm{C}$ and $60{ }^{\circ} \mathrm{C}$. Inset: Arrhenius plots for conductivity of dEAN and PEGDA/dEAN crosslinked ionogels at the indicated compositions. Solid straight lines are fits to Arrhenius equation (Eqn 5) using the parameters shown in Table 1 . 


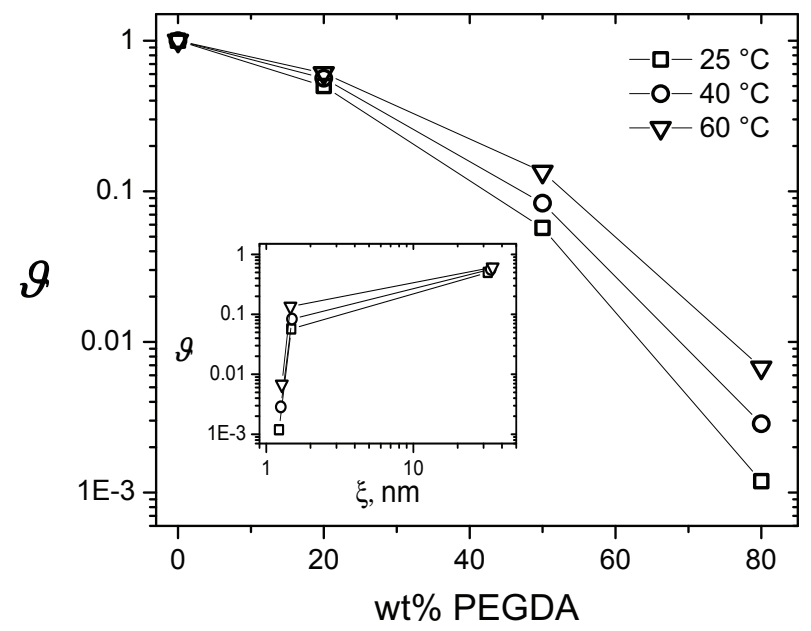

Figure 8: Obstruction factor $(\vartheta)$ as a function of concentration and temperature for PEGDA/dEAN crosslinked ionogels. Inset: Obstruction factor versus network mesh size. The symbols for both the main plot and the inset plot correspond to the indicated temperatures.

$$
\vartheta \equiv \frac{\sigma}{(1-\phi) \sigma_{d E A N}}
$$

where $\phi$ is the volume fraction and the term $(1-\phi)$ accounts for the fact that the effective volume for ion transport is the volume of dEAN alone. Figure 8 shows the obstruction factor as a function of [PEGDA] at $25^{\circ} \mathrm{C}, 40{ }^{\circ} \mathrm{C}$ and $60{ }^{\circ} \mathrm{C}$. The value of $\vartheta$ decrease two to three orders of magnitude in the composition range $[\mathrm{PEGDA}] \leq 80 \mathrm{wt} \%$. As expected, the greatest change is observed at the lowest temperature, as a result of the lowest ion mobility.

The obstruction factor is directly related to the network mesh size $(\xi)$. The inset in Figure 8 shows a sharp increase in $\vartheta$ at $\xi$ values near $1 \mathrm{~nm}$, whereas the $\vartheta$ dependence on $\xi$ is weaker at higher mesh sizes. This behavior can be due to two effects. One is binding of EAN ions to the polymer network via hydrogen bonding, $[33,34,35]$ which would reduce the molecular mobility and hence the conductivity. This effect may be considerable at high polymer compositions, i.e, when the ratio between EAN molecules and binding sites is low. Moreover, the decreasing strength of H-bonds with temperature $[36,37$ can explain the lower decrease in $\vartheta$ at higher temperatures. The second possible reason for the sharp decrease in $\vartheta$ is that, at high polymer concentrations, the network mesh size $(\xi \sim 1 \mathrm{~nm})$ is comparable 
to the characteristic size of the sponge-like nanostructure inherent to pure EAN $(\sim 0.97 \mathrm{~nm})[25]$. Therefore, at concentrations [PEGDA] $>50 \mathrm{wt} \%$, the EAN structure may be disrupted and the ion transport may be seriously penalized, as indicated by the high activation energy (see Table 1).

\section{CONCLUSIONS}

We have synthesized crosslinked ionogels (x-IGs) based on a protic ionic liquid (EAN) and a bifunctional polyoxyethylene (PEGDA) via UV-initiated radical reactions, in the entire range of compositions. Mechanical and dielectric properties of the $\mathrm{x}$-IGs were investigated in a wide temperature range. Both, the ionic conductivity and the shear modulus are directly correlated with the network mesh size ( $\xi$, measured by SANS). The shear modulus fol-

lows the expected power law dependence with mesh size, $G \sim \xi^{-3}$, whereas the obstruction factor increase monotonically with $\xi$, showing a sharp increase when $\xi$ is comparable to the size of the EAN nanostructure.

This study provides a simple route to prepare solid electrolytes with tunable nanostructure, mechanical properties and dielectric behavior, which can be potentially useful in electrochemical cell applications (e.g., in batteries, fuel cells, gas sensors, etc. [9]). One may readily envision additional microstructure tunability by varying the molecular weight of the polymer or by providing solvophobicity to the polymer, e.g., by attaching a solvophobic block to the solvophilic PEGDA chain, or by using commercial PEO-PPOPEO triblock copolymers (Pluronics). The latter approach is currently being explored by us [38].

\section{Acknowledgments}

We wish to thank Professor N. J. Wagner (University of Delaware) for the use of the facilities at the Center for Neutron Science at the University of Delaware and for helpful comments. P.J.B acknowledges support from the U.S. Department of Energy, Office of Basic Energy Sciences, Division of Materials Sciences and Engineering under Award No. DE-FG02-09ER46626. The authors acknowledge the HANARO Center of the Korea Atomic Energy Research Institute for providing access to the 40m SANS instrument used in this work. MJL is supported by the National Research Foundation of Korea (No. 2011-0031931). 
[1] T. Welton, Room-temperature ionic liquids. solvents for synthesis and catalysis, Chem. Rev. 99 (1999) 2071-2084.

[2] W. Xu, C. A. Angell, Solvent-free electrolytes with aqueous solution-like conductivities, Science 302 (2003) 422-425.

[3] H. Ohno, Electrochemical aspects of ionic liquids, Wiley-Interscience, Hoboken, N.J., 2005.

[4] P. Snedden, A. I. Cooper, K. Scott, N. Winterton, Cross-linked polymerionic liquid composite materials, Macromolecules 36 (2003) 45494556.

[5] P. Scovazzo, J. Kieft, D. A. Finan, C. Koval, D. DuBois, R. Noble, Gas separations using non-hexafluorophosphate [pf6] anion supported ionic liquid membranes, J. Membrane Sci. 238 (2004) 57-63.

[6] J. Tang, W. Sun, H. Tang, M. Radosz, Y. Shen, Enhanced co2 absorption of poly(ionic liquid)s, Macromolecules 38 (2005) 2037-2039.

[7] M. L. Hoarfrost, R. A. Segalman, Ionic conductivity of nanostructured block copolymer/ionic liquid membranes, Macromolecules 44 (2011) $5281-5288$.

[8] K. H. Lee, S. Zhang, T. P. Lodge, C. D. Frisbie, Electrical impedance of spin-coatable ion gel films, J. Phys. Chem. B 115 (2011) 3315-3321.

[9] J. L. Bideau, L. Viau, A. Vioux, Ionogels, ionic liquid based hybrid materials, Chem. Soc. Rev. 40 (2011) 907-925.

[10] Y. He, T. P. Lodge, Thermoreversible ion gels with tunable melting temperatures from triblock and pentablock copolymers, Macromolecules 41 (2008) 167-174.

[11] T. P. Lodge, A unique platform for materials design, Science 321 (2008) $50-51$.

[12] S. Zhang, K. H. Lee, J. Sun, C. D. Frisbie, T. P. Lodge, Viscoelastic properties, ionic conductivity, and materials design considerations for poly(styrene-b-ethylene oxide-b-styrene)-based ion gel electrolytes, Macromolecules 44 (2011) 8981-8989. 
[13] M. A. Klingshirn, S. K. Spear, R. Subramanian, J. D. Holbrey, J. G. Huddleston, R. D. Rogers, Gelation of ionic liquids using a cross-linked poly(ethylene glycol) gel matrix, Chem. Mater. 16 (2004) 3091-3097.

[14] M. A. B. H. Susan, T. Kaneko, A. Noda, M. Watanabe, Ion gels prepared by in situ radical polymerization of vinyl monomers in an ionic liquid and their characterization as polymer electrolytes, J. Am. Chem. Soc. 127 (2005) 4976-4983.

[15] D. M. Tigelaar, M. A. B. Meador, W. R. Bennett, Composite electrolytes for lithium batteries: ionic liquids in aptes cross-linked polymers, Macromolecules 40 (2007) 4159-4164.

[16] K. Matsumoto, T. Endo, Confinement of ionic liquid by networked polymers based on multifunctional epoxy resins, Macromolecules 41 (2008) 6981-6986.

[17] B. Rupp, M. Schmuck, A. Balducci, M. Winter, W. Kern, Polymer electrolyte for lithium batteries based on photochemically crosslinked poly(ethylene oxide) and ionic liquid, Eur. Polym. J. 44 (2008) 29862990 .

[18] E. S. Sterner, Z. P. Rosol, E. M. Gross, S. M. Gross, Thermal analysis and ionic conductivity of ionic liquid containing composites with different crosslinkers, J. App. Polym. Sci. 114 (2009) 2963-2970.

[19] C. A. Angell, N. Byrne, J.-P. Belieres, Parallel developments in aprotic and protic ionic liquids: Physical chemistry and applications, Acc. Chem. Res. 40 (2007) 1228-1236.

[20] C. R. López-Barrón, M. G. Basavaraj, L. DeRita, N. J. Wagner, Spongeto-lamellar transition in a double-tail cationic surfactant/protic ionic liquid system: Structural and rheological analysis, J. Phys. Chem. B 116 (2012) 813-822.

[21] A. D. Hollingsworth, D. A. Saville, A broad frequency range dielectric spectrometer for colloidal suspensions: cell design, calibration, and validation, J. Coll. Int. Sci. 257 (2003) 65-76.

[22] P. C. Hiemenz, T. Lodge, Polymer chemistry, 2nd Edition, CRC Press, Boca Raton, FL, 2007. 
[23] M. E. Seitz, W. R. Burghardt, K. T. Faber, K. R. Shull, Self-assembly and stress relaxation in acrylic triblock copolymer gels, Macromolecules 40 (2007) 1218-1226.

[24] F. Tanaka, S. F. Edwards, Viscoelastic properties of physically crosslinked networks: Part 2. dynamic mechanical moduli, J. NonNewtonian Fluid Mech. 43 (1992) 273-288.

[25] R. Atkin, G. G. Warr, The smallest amphiphiles: Nanostructure in protic room-temperature ionic liquids with short alkyl groups, J. Phys. Chem. B 112 (2008) 4164-4166.

[26] J. Bastide, S. J. Candau, Structure of Gels as Investigated by Means of Static Scattering Techniques, Physical Properties of Polymeric Gels, John Wiley and Sons Ltd., Chichester ; New York, 1996, Ch. 5, pp. 143-308.

[27] M. Rubinstein, R. H. Colby, Polymer physics, Oxford University Press, Oxford ; New York, 2003.

[28] K. A. Erk, J. D. Martin, Y. T. Hu, K. R. Shull, Extreme strain localization and sliding friction in physically associating polymer gels, Langmuir 28 (2012) 4472-4478.

[29] A. D. Hollingsworth, D. A. Saville, Dielectric spectroscopy and electrophoretic mobility measurements interpreted with the standard electrokinetic model, J. Coll. Int. Sci. 272 (2004) 235-245.

[30] F. Kremer, A. Schönhals, Broadband dielectric spectroscopy, Springer, Berlin ; New York, 2003.

[31] H. Weingartner, A. Knocks, W. Schrader, U. Kaatze, Dielectric spectroscopy of the room temperature molten salt ethylammonium nitrate, J. Phys. Chem. A 105 (2001) 8646-8650.

[32] R. Strey, R. Schomacker, D. Roux, F. Nallet, U. Olsson, Dilute lamellar and 13 phases in the binary water-c12e5 system, J. Chem. Soc., Faraday Trans. 86 (1990) 2253-2261.

[33] O. Werzer, G. G. Warr, R. Atkin, Conformation of poly(ethylene oxide) dissolved in ethylammonium nitrate, J. Phys. Chem. B 115 (2011) 648652 . 
[34] J. Hunger, T. Sonnleitner, L. Liu, R. Buchner, M. Bonn, H. J. Bakker, Hydrogen-bond dynamics in a protic ionic liquid: Evidence of largeangle jumps, J. Phys. Chem. Lett. 3 (2012) 3034-3038.

[35] T. L. Greaves, C. J. Drummond, Protic ionic liquids: Evolving structure??property relationships and expanding applications, Chem. Rev. 115 (2015) 11379-11448.

[36] S. Lusse, K. Arnold, The interaction of poly(ethylene glycol) with water studied by $1 \mathrm{~h}$ and $2 \mathrm{~h}$ nmr relaxation time measurements, Macromolecules 29 (1996) 4251-4257.

[37] G. D. Smith, D. Bedrov, Roles of enthalpy, entropy, and hydrogen bonding in the lower critical solution temperature behavior of poly(ethylene oxide)/water solutions, J. Phys. Chem. B 107 (2003) 3095-3097.

[38] C. R. López-Barrón, D. Li, N. J. Wagner, J. L. Caplan, Triblock copolymer self-assembly in ionic liquids: Effect of peo block length on the selfassembly of peo-ppo-peo in ethylammonium nitrate, Macromolecules 47 (2014) 7484-7495. 